\title{
Jakub Beně̌
}

Projective deformation of line congruences in five-dimensional projective spaces

Czechoslovak Mathematical Journal, Vol. 18 (1968), No. 3, 457-475

Persistent URL: http://dml.cz/dmlcz/100846

\section{Terms of use:}

(C) Institute of Mathematics AS CR, 1968

Institute of Mathematics of the Czech Academy of Sciences provides access to digitized documents strictly for personal use. Each copy of any part of this document must contain these Terms of use.

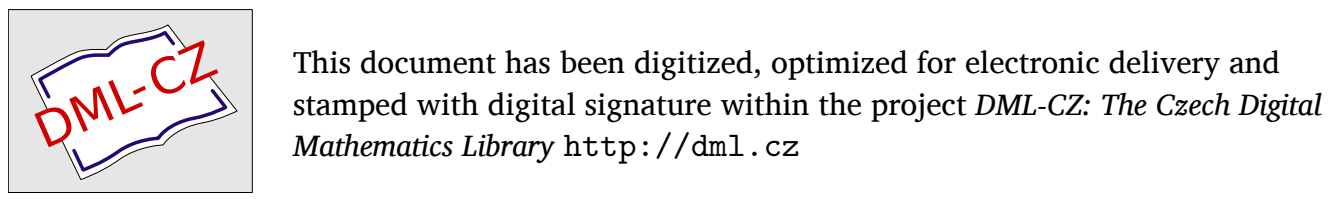




\title{
PROJECTIVE DEFORMATION OF LINE CONGRUENCES IN FIVE-DIMENSIONAL PROJECTIVE SPACES
}

\author{
JAKUB Beneš, Brno
}

(Received January 11, 1967)

\begin{abstract}
A. ŠvEC considered a projective deformation of the second order of line congruences in $n$-dimensional projective spaces for the first time in his paper [1] and has proved there that this problem is for $n \geqq 5$ equivalent to a point deformation. Later he has solved completely the problem of projective deformation of $(m+1)$ st order of surfaces with conjugate net in $(2 m+1)$-dimensional projective space $([3])$. The essential results achieved in this book allow us to expect that the projective deformation of $(m+1)$ st order of line congruences in $(2 m+1)$-projective spaces yields substantial results, too. The following solution of this problem for $m=2$ indicates this and together with the well known case $m=1$ facilitates a predetermination of the situation in the general case.
\end{abstract}

\section{SPECIALIZATION OF FRAMES AND FUNDAMENTAL EQUATIONS OF CONGRUENCE}

Let us consider a congruence $L$ in a 5 -dimensional projective space with character $\operatorname{dim} \tau=3(\tau$ is a tangent space of the congruence $L$ at its generating line $p$ ) and assume that $L$ is non-parabolic with two focal surfaces; hence $L$ has two systems of developable surfaces. With each line $p(u, v)$, where the admissible pairs $(u, v)$ are taken from an open neighbourhood of $C^{2}(C=$ complex numbers), we associate a frame $R$ consisting of linearly independent points $A_{1}, A_{2}, \ldots, A_{6}$ so that

$$
\left[A_{1} A_{2} \ldots A_{6}\right]=1
$$

and $\left(A_{1}, A_{2}\right)=p$.

The fundamental equations of the moving frame and structure equations are

$$
\mathrm{d} A_{i}=\sum_{j=1}^{6} \omega_{i j} A_{j}, \quad \sum_{i=1}^{6} \omega_{i i}=0, \quad \mathrm{~d} \omega_{i j}=\sum_{k=1}^{6} \omega_{i k} \wedge \omega_{k j} .
$$


Let us specialize the frames of the congruence $L$ according to A. Švec [1] p. 31-34. By means of the well known Cartan's process it is possible to carry out a further specialization of frames so that the congruence $L$ is given by a closed system

$$
\begin{array}{ll}
\omega_{12}=\alpha_{1} \omega_{2}, & \omega_{14}=\omega_{15}=\omega_{16}=0, \\
\omega_{21}=\alpha_{2} \omega_{1}, & \omega_{23}=\omega_{25}=\omega_{26}=0, \\
\omega_{35}=\omega_{1}, & \omega_{32}=\omega_{34}=\omega_{36}=0, \\
\omega_{46}=\omega_{2}, & \omega_{41}=\omega_{43}=\omega_{45}=0, \\
\omega_{56}=\beta_{1} \omega_{2}, & \omega_{54}=0, \quad \omega_{65}=\beta_{2} \omega_{1}, \quad \omega_{63}=0
\end{array}
$$

$$
\begin{aligned}
\omega_{2} & \wedge\left\{\mathrm{d} \alpha_{1}-\alpha_{1}\left(\omega_{44}+\omega_{11}-2 \omega_{22}\right)\right\}=0, \\
\omega_{1} & \wedge\left\{\mathrm{d} \alpha_{2}-\alpha_{2}\left(\omega_{33}+\omega_{22}-2 \omega_{11}\right)\right\}=0, \\
\omega_{1} & \wedge\left(2 \omega_{33}-\omega_{11}-\omega_{55}\right)=0, \\
\beta_{2} \omega_{1} & \wedge \omega_{64}-\omega_{2} \wedge \omega_{52}=0, \\
\omega_{1} & \wedge \omega_{52}-\alpha_{1} \omega_{2} \wedge \omega_{31}=0, \\
\omega_{2} & \wedge\left\{\mathrm{d} \beta_{1}-\beta_{1}\left(2 \omega_{66}-\omega_{55}-\omega_{44}\right)\right\}=0, \\
\omega_{1} & \wedge\left\{\mathrm{d} \beta_{2}-\beta_{2}\left(2 \omega_{55}-\omega_{66}-\omega_{33}\right)\right\}=0, \\
\omega_{2} & \wedge\left(2 \omega_{44}-\omega_{22}-\omega_{66}\right)=0, \\
\alpha_{2} \omega_{1} & \wedge \omega_{42}-\omega_{2} \wedge \omega_{61}=0, \\
\omega_{1} & \wedge \omega_{61}-\beta_{1} \omega_{2} \wedge \omega_{53}=0,
\end{aligned}
$$

where

$$
\omega_{1}=\omega_{13}, \omega_{2}=\omega_{24} \text { and } \omega_{1} \wedge \omega_{2} \neq 0
$$

By this specialization of frames the equations of developable surfaces are $\omega_{1} \omega_{2}=0$; $A_{1}, A_{2}$ are foci on $p, p_{1}=\left(A_{1}, A_{3}\right)\left\{p_{-1}=\left(A_{2}, A_{4}\right)\right\}$ is the first \{minus first Laplace transform $L_{1}\left\{L_{-1}\right\}$ of congruence $L$ in the direction $\omega_{2}=0\left\{\omega_{1}=0\right\}$, $A_{3}\left\{A_{4}\right\}$ is the second focus on $p_{1}\left\{p_{-1}\right\}$. The fundamental equations of congruence $L$ are given by $(2,2)$ and (3). The basic invariant forms of congruence $L$ are (see [3], p. 35):

$$
\begin{aligned}
& \varphi=\alpha_{1} \alpha_{2} \omega_{1} \omega_{2} \text { (point form), } \\
& \varphi^{*}=\beta_{1} \beta_{2} \omega_{1} \omega_{2} \text { (hyperplanar form), } \\
& F_{1}=\alpha_{1} \beta_{1} \frac{\omega_{2}^{4}}{\omega_{1}^{2}}, \quad F_{2}=\alpha_{2} \beta_{2} \frac{\omega_{1}^{4}}{\omega_{2}^{2}} \quad \text { (focal forms), } \\
& G_{1}=\frac{\alpha_{1}}{\beta_{2}}\left(\frac{\omega_{2}}{\omega_{1}}\right)^{3}, \quad G_{2}=\frac{\alpha_{2}}{\beta_{1}}\left(\frac{\omega_{1}}{\omega_{2}}\right)^{3} \quad \text { (quasi-asymptotic forms). }
\end{aligned}
$$




\section{DUALIZATION OF CONGRUENCE $L$}

Let $P_{5}^{*}$ be the dual space to the space $P_{5}$. To the frame $R$ in $P_{5}$, we introduce the dual frame $R^{*}$ formed by analytical hyperplanes

$$
E_{k}=(-1)^{k+1}\left[A_{1}, \ldots, A_{k-1}, A_{k}, \ldots, A_{6}\right] \quad(k=1,2, \ldots, 6) .
$$

The tangent space $\tau=\left(A_{1}, A_{2}, A_{3}, A_{4}\right)$ of $L$ at the line $p$ describes a line $p^{*}=\left(E_{5}, E_{6}\right)$ in the dual space $P_{5}^{*}$ (see [2], p. 91) generating the dual congruence $L^{*}$, which is called the dualization of the congruence $L$.

If $\left(2_{1,2}\right)$ and (3) are fundamental equations of congruence $L$, then

$$
\mathrm{d} E_{i}=-\sum_{j=1}^{6} \omega_{j i} E_{j}
$$

together with (3) are the fundamental equations of congruence $L^{*}$. In the following we shall suppose that the congruence $L^{*}$ has two focal surfaces as the congruence $L$ has, i.e.

$$
\alpha_{1} \alpha_{2} \beta_{1} \beta_{2} \neq 0 \text {. }
$$

From the fundamental equations $\left(2_{1,2}\right),(3)$ it results that the hyperplane $E_{5}\left\{E_{6}\right\}$ is the osculating space of the focal surface $\left(A_{1}\right)\left\{\left(A_{2}\right)\right\}$ at the point $A_{1}\left\{A_{2}\right\}$; we shall call it the focal hyperplane.

The focal hyperplanes are points in the dual space $P_{5}^{*}$ and generate the focal surfaces $\left(E_{5}\right),\left(E_{6}\right)$ of the congruence $L^{*}$. If $S$ is the developable surface of $L$ with its edge placed on the focal surface $\left(A_{1}\right)$, then the corresponding developable surface $S^{*}$ in $L^{*}$ has its edge placed on the focal surface $\left(E_{5}\right)$. If we make an agreement that $A_{1}$ is the first and $A_{2}$ is the second focus on $p$, then the natural developable correspondence $L \rightarrow L^{*}$ transfers this orientation on the congruence $L^{*}$ such that $E_{5}$ is the first and $E_{6}$ is the second focus on $p^{*}$. So we have the following substitute if passing from $L$ to $L^{*}$,

$$
\left|\begin{array}{l:lllllll}
L: A_{i} \omega_{j k} & i & 1 & 2 & 3 & 4 & 5 & 6 \\
L^{*}: E_{i} \omega_{j^{*}} & i^{*} & 5 & 6 & 3 & 4 & 2 & 1
\end{array}\right|
$$

The left-hand side of the table indicates the correspondence between points and hyperplanes and between relative components of frames $R$ and $R^{*}$, the right-hand side shows the changing of indices.

The other expressions are changed according to the following diagram

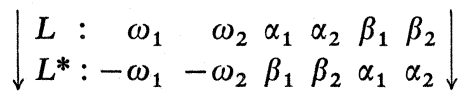




\section{DEVELOPABLE CORRESPONDENCE}

Let $L^{\prime}$ be another non-parabolic line congruence immersed in a projective space $P_{5}^{\prime}$; we denote all expressions related to $L^{\prime}$ by an apostrophe. Let the frames associated with $L^{\prime}$ be specialized in the same way as those associated with $L$; hence $\left(1^{\prime}\right)$ through $\left(11^{\prime}\right)$ hold.

Let $C: L \rightarrow L^{\prime}$ be a one-to-one correspondence associating a line $p^{\prime} \in L^{\prime}$ with a line $p \in L$. In the following development we shall mostly consider developable correspondences; that is why we deduce some useful expressions for this kind of correspondences.

Without loss of generality, we may write the equations of the developable correspondence in the form

$$
\omega_{1}^{\prime}=\omega_{1}, \quad \omega_{2}^{\prime}=\omega_{2}
$$

or

$$
\tau_{13}=0, \quad \tau_{24}=0
$$

if denoting

$$
\tau_{i j}=\omega_{i j}^{\prime}-\omega_{i j}
$$

Using exterior differentiation of (12), Cartan's lemma and the equations (4), (4'), (12), (13), we get the relations

$$
\begin{array}{ll}
\tau_{33}-\tau_{11}=s_{1} \omega_{1}, & \tau_{44}-\tau_{22}=s_{2} \omega_{2}, \\
\tau_{55}-\tau_{33}=s_{3} \omega_{1}, & \tau_{66}-\tau_{44}=s_{4} \omega_{2},
\end{array}
$$

the exterior differentiations of which are

$$
\begin{aligned}
& \omega_{1} \wedge\left\{\mathrm{d} s_{1}-s_{1}\left(\omega_{33}-\omega_{11}\right)+\tau_{53}-2 \tau_{31}\right\}+\left(\alpha_{1}^{\prime} \alpha_{2}^{\prime}-\alpha_{1} \alpha_{2}\right) \omega_{1} \wedge \omega_{2}=0 \\
& \omega_{2} \wedge\left\{\mathrm{d}_{2}-s_{2}\left(\omega_{44}-\omega_{22}\right)+\tau_{64}-2 \tau_{42}\right\}-\left(\alpha_{1}^{\prime} \alpha_{2}^{\prime}-\alpha_{1} \alpha_{2}\right) \omega_{1} \wedge \omega_{2}=0 \\
& \omega_{1} \wedge\left\{\mathrm{d} s_{3}-s_{3}\left(\omega_{33}-\omega_{11}\right)-2 \tau_{53}+\tau_{31}\right\}+\left(\beta_{1}^{\prime} \beta_{2}^{\prime}-\beta_{1} \beta_{2}\right) \omega_{1} \wedge \omega_{2}=0 \\
& \omega_{2} \wedge\left\{\mathrm{d} s_{4}-s_{4}\left(\omega_{44}-\omega_{22}\right)-2 \tau_{64}+\tau_{42}\right\}-\left(\beta_{1}^{\prime} \beta_{2}^{\prime}-\beta_{1} \beta_{2}\right) \omega_{1} \wedge \omega_{2}=0
\end{aligned}
$$

Further, from the equations $(4),\left(4^{\prime}\right),(12),(13)$, we obtain

$$
\begin{aligned}
& \mathrm{d} \frac{\alpha_{1}^{\prime}}{\alpha_{1}}-\frac{\alpha_{1}^{\prime}}{\alpha_{1}}\left(\tau_{11}-\tau_{22}\right)=m_{1} \omega_{2}, \quad \mathrm{~d} \frac{\alpha_{2}^{\prime}}{\alpha_{2}}-\frac{\alpha_{2}^{\prime}}{\alpha_{2}}\left(\tau_{22}-\tau_{11}\right)=m_{2} \omega_{1}, \\
& \mathrm{~d} \frac{\beta_{1}^{\prime}}{\beta_{1}}-\frac{\beta_{1}^{\prime}}{\beta_{1}}\left(\tau_{66}-\tau_{55}\right)=n_{1} \omega_{2}, \quad \mathrm{~d} \frac{\beta_{2}^{\prime}}{\beta_{2}}-\frac{\beta_{2}^{\prime}}{\beta_{2}}\left(\tau_{55}-\tau_{66}\right)=n_{2} \omega_{1} .
\end{aligned}
$$

The tables (10), (11) and the equations (14), (16) yield the following substitution if 
passing from congruences $L, L^{\prime}$ in developable correspondence $C$ to the dual congruences $L^{*}, L^{*}$ in the developable correspondence $C^{*}$

$$
\left|\begin{array}{lrrrrrrrr}
C: L \rightarrow L^{\prime} & s_{1} & s_{2} & s_{3} & s_{4} & m_{1} & m_{2} & n_{1} & n_{2} \\
C^{*}: L^{*} \rightarrow L^{\prime *} & -s_{3} & -s_{4} & -s_{1} & -s_{2} & -n_{1} & -n_{2} & -m_{1} & -m_{2}
\end{array}\right|
$$

\section{PROJECTIVE DEFORMATION OF THE THIRD ORDER}

It is well known that a line $p$ of the projective space $P_{5}$ can be represented as a point of the Grassmann manifold of dimension eight in the 14-dimensional projective space $g\left(P_{5}\right)$. The image of the congruence $L$ in $P_{5}$ is a surface $g(L)$ in $g\left(P_{5}\right)$. Let us denote $[A, B]$ the Grassmann coordinates of the line $(A, B)$. If $A_{i}$ is the frame in $P_{5}$, then $\left[A_{i}, A_{j}\right]$ form the frame in $g\left(P_{5}\right)$. Let $K: P_{5} \rightarrow P_{5}^{\prime}$ be a collineation given by the equations

$$
K A_{i}=\sum_{j=1}^{6} \alpha_{i j} A_{j}^{\prime} \quad(i=1,2, \ldots, 6)
$$

then $g(K): g\left(P_{5}\right) \rightarrow g\left(P_{5}^{\prime}\right)$ given by

$$
K\left[A_{i}, A_{j}\right]=\sum_{k, l=1}^{6} \alpha_{i k} \alpha_{j l}\left[A_{k}^{\prime}, A_{l}^{\prime}\right] \quad(i, j=1,2, \ldots, 6, i \neq j)
$$

is an induced collineation.

In accordance with A. Švec (see [3], p. 17) we have the following definition.

Definition 1. Let $C: L \rightarrow L^{\prime}$ be a correspondence. We call $C$ the projective deformation of the third order, if for each line $p \in L$ there exists a collineation $K: P_{5} \rightarrow P_{5}^{\prime}$ such that the surfaces $g(K) g(L)$ and $g\left(L^{\prime}\right)$ have an analytic contact of order three at the point $g\left(p^{\prime}\right)$. We shall say that the collineation $K$ (s.c. osculating collineation of the third order) realizes the projective deformation $C$.

\section{ANALYTICAL CONDITIONS FOR PROJECTIVE DEFORMATION}

From Definition 1 it follows that a correspondence $C: L \rightarrow L^{\prime}$ is a projective deformation of the third order if there exists a collineation $K$ given by the equations (18) such that

$$
K \mathrm{~d}^{m}\left[A_{1}, A_{2}\right]=\sum_{k=0}^{m}\left(\begin{array}{l}
m \\
k
\end{array}\right) \vartheta_{k} \mathrm{~d}^{m-k}\left[A_{1}^{\prime}, A_{2}^{\prime}\right] \quad(m=0,1,2,3),
$$

where $\vartheta_{k}$ is an appropriate $k$-form. 
According to [3] p. 18-20, the necessary and sufficient conditions for (19) to be fulfilled in the cases $m=0,1,2$ are as follows: $C$ is developable and

$$
\varrho^{2} \alpha_{2}=\alpha_{2}^{\prime}, \quad \varrho^{-2} \alpha_{1}=\alpha_{1}^{\prime} .
$$

The most general osculating collineation realizing a projective deformation of the second order is given by

$$
\begin{gathered}
K A_{1}=\varrho A_{1}^{\prime}, \quad K A_{2}=\varrho^{-1} A_{2}^{\prime}, \quad K A_{3}=\alpha_{31} A_{1}^{\prime}+\varrho A_{3}^{\prime}, \\
K A_{4}=\alpha_{42} A_{2}^{\prime}+\varrho^{-1} A_{2}^{\prime}, \quad K A_{5}=\alpha_{51} A_{1}^{\prime}+\alpha_{52} A_{2}^{\prime}+\alpha_{53} A_{3}^{\prime}+\varrho A_{5}^{\prime}, \\
K A_{6}=\alpha_{61} A_{1}^{\prime}+\alpha_{62} A_{2}^{\prime}+\alpha_{64} A_{4}^{\prime}+\varrho^{-1} A_{6}^{\prime} \\
\alpha_{53}-2 \alpha_{31}=\varrho s_{1}, \quad \alpha_{64}-2 \alpha_{42}=\varrho^{-1} s_{2} \\
(22) \quad \tau_{22}+\varrho^{-1} \alpha_{31} \omega_{1}+\varrho \omega_{42} \omega_{2} \\
\vartheta_{1}=-\tau_{11}-\tau_{2}=-\mathrm{d}\left(\tau_{11}+\tau_{22}\right)+\left(\tau_{11}+\tau_{22}\right)^{2}-\omega_{1} \tau_{31}-\omega_{2} \tau_{42}+\varrho^{-1} \alpha_{51} \omega_{1}^{2}+ \\
+\varrho \alpha_{62} \omega_{2}^{2}+2 \alpha_{31} \alpha_{42} \omega_{1} \omega_{2}+\varrho^{-1} \alpha_{31}\left\{\mathrm{~d} \omega_{1}-\omega_{1}\left(2 \tau_{11}+2 \tau_{22}+\right.\right. \\
\left.\left.+\omega_{11}-\omega_{33}\right)\right\}+\varrho \alpha_{42}\left\{\mathrm{~d} \omega_{2}-\omega_{2}\left(2 \tau_{11}+2 \tau_{22}+\omega_{22}-\omega_{44}\right)\right\} .
\end{gathered}
$$

The following relations (24), (25) may be checked by a direct computation if the equations $(2,2),(3),\left(2_{1,2}^{\prime}\right),\left(3^{\prime}\right),(12),(13),(14),(20),(21),(22),(23)$ are used;

$$
\begin{gathered}
K \mathrm{~d}^{3}\left[A_{1}, A_{2}\right]-\mathrm{d}^{3}\left[A_{1}^{\prime}, A_{2}^{\prime}\right]-3 \vartheta_{1} \mathrm{~d}^{2}\left[A_{1}^{\prime}, A_{2}^{\prime}\right]-3 \vartheta_{2} \mathrm{~d}\left[A_{1}^{\prime}, A_{2}^{\prime}\right]= \\
=\sum_{(i k)} \Phi_{i k}\left[A_{i}^{\prime}, A_{k}^{\prime}\right],
\end{gathered}
$$

where

(25)

$$
\begin{aligned}
\Phi_{13}= & -\varrho^{-1} \alpha_{2}^{\prime} \alpha_{31} \omega_{1}^{3}+3 \varrho \alpha_{2}^{\prime} \alpha_{42} \omega_{1}^{2} \omega_{2}-3 \varrho \alpha_{61} \omega_{1} \omega_{2}^{2}+\varrho \beta_{1} \alpha_{53} \omega_{2}^{3}, \\
\Phi_{24}= & \varrho \alpha_{1}^{\prime} \alpha_{42} \omega_{2}^{3}-3 \varrho^{-1} \alpha_{1}^{\prime} \alpha_{31} \omega_{1} \omega_{2}^{2}+3 \varrho \alpha_{52} \omega_{1}^{2} \omega_{2}-\varrho^{-1} \beta_{2} \alpha_{64} \omega_{1}^{3}, \\
\Phi_{15}= & \left(\varrho^{2} \beta_{1}-\beta_{1}^{\prime}\right) \omega_{2}^{3}, \quad \Phi_{26}=\left(\beta_{2}^{\prime}-\varrho^{-2} \beta_{2}\right) \omega_{1}^{3}, \\
\Phi_{16}= & -\left(3 \varrho \alpha_{42}+2 s_{2}+s_{4}\right) \omega_{2}^{3}, \quad \Phi_{25}=\left(3 \varrho \alpha_{31}+2 s_{1}+s_{3}\right) \omega_{1}^{3}, \\
\Phi_{14}= & -\left\{\mathrm{d} s_{2}-s_{2}\left(\omega_{44}-\omega_{22}\right)+\tau_{64}-2 \tau_{42}\right\} \omega_{2}^{2}-\varrho \alpha_{42} \omega_{2}^{2}\left\{3 s_{2} \omega_{2}+\right. \\
& \left.+2\left(2 \omega_{44}-\omega_{66}-\omega_{22}\right)\right\}-\left(3 \varrho \alpha_{62}+s_{2}^{2}\right) \omega_{2}^{3}-s_{2} \omega_{2}^{2}\left(2 \omega_{44}-\omega_{66}-\omega_{22}\right), \\
& \left\{\mathrm{d} s_{1}-s_{1}\left(\omega_{33}-\omega_{11}\right)+\tau_{53}-2 \tau_{31}\right\} \omega_{1}^{2}+\varrho^{-1} \alpha_{31} \omega_{1}^{2}\left\{3 s_{1} \omega_{1}+\right. \\
& \left.+2\left(2 \omega_{33}-\omega_{55}-\omega_{11}\right)\right\}+\left(3 \varrho^{-1} \alpha_{51}+s_{1}^{2}\right) \omega_{1}^{3}+s_{1} \omega_{1}^{2}\left(2 \omega_{33}-\omega_{55}-\omega_{11}\right), \\
\Phi_{23}= & \Phi_{35} \equiv \Phi_{45} \equiv \Phi_{46} \equiv \Phi_{56} \equiv 0 .
\end{aligned}
$$

The coefficient $\Phi_{12}$ does not interest us.

Now, from $(19, m=3),(20),(21),(22),(24)$ and with respect to (9) we get the necessary and sufficient analytic conditions for $C$ to be the projective deformation of the third order, which we formulate in 
Proposition 1. The developable correspondence $C: L \rightarrow L^{\prime}$ is a projective deformation of the third order if and only if there exists $\varrho \neq 0$ such that

$$
\alpha_{1}^{\prime}=\varrho^{-2} \alpha_{1}, \quad \alpha_{2}^{\prime}=\varrho^{2} \alpha_{2}, \quad \beta_{1}^{\prime}=\varrho^{2} \beta_{1}, \quad \beta_{2}^{\prime}=\varrho^{-2} \beta_{2}
$$

and

$$
s_{1}=s_{2}=s_{3}=s_{4}=0 .
$$

The most general osculating collineation realizing this deformation is given by the equations

$$
\begin{gathered}
K A_{1}=\varrho A_{1}^{\prime}, \quad K A_{2}=\varrho^{-1} A_{2}^{\prime}, \quad K A_{3}=\varrho A_{3}^{\prime}, \quad K A_{4}=\varrho^{-1} A_{4}^{\prime}, \\
K A_{5}=\alpha_{51} A_{1}^{\prime}+\varrho A_{5}^{\prime}, \quad K A_{6}=\alpha_{62} A_{2}^{\prime}+\varrho^{-1} A_{6}^{\prime},
\end{gathered}
$$

where

$$
3 \varrho^{-1} \alpha_{51} \omega_{1}=2 \tau_{31}-\tau_{53}, \quad 3 \varrho \alpha_{62} \omega_{2}=2 \tau_{42}-\tau_{64} .
$$

A comparing of $(26),(6),\left(6^{\prime}\right),(12)$ and $[2](p .97,98)$ yields the following assertion: If the correspondence $C: L \rightarrow L^{\prime}$ is a projective deformation of the third order, then

$$
\varphi=\varphi^{\prime}, \quad \varphi^{*}=\varphi^{*}, \quad G_{1}=G_{1}^{\prime}, \quad G_{2}=G_{2}^{\prime}, \quad F_{1}=F_{1}^{\prime}, \quad F_{2}=F_{2}^{\prime} .
$$

This result can be summarized in (see [2] p. 97, 98 and [3] p. 39).

Proposition 2. Let a correspondence $C: L \rightarrow L^{\prime}$ be a projective deformation of the third order. Then the correspondence $C$ is

1. point deformation,

2. hyperplanar deformation,

3. quasiasymptotic deformation of the first and second kind,

4. focal deformation of the first and second kind,

5. projective bideformation of the second order.

\section{PROJECTIVE DEFORMATION OF DUAL CONGRUENCES}

The following necessary and sufficient conditions for the induced developable correspondence $C^{*}: L^{*} \rightarrow L^{*}$ to be a projective deformation of the third order are easily verified by using (10), (11), (17);

$$
\begin{aligned}
\beta_{1}^{\prime}=\sigma^{-2} \beta_{1}, \quad \beta_{2}^{\prime} & =\sigma^{2} \beta_{2}, \quad \alpha_{1}^{\prime}=\sigma^{2} \alpha_{1}, \quad \alpha_{2}^{\prime}=\sigma^{-2} \alpha_{2}, \\
s_{1} & =s_{2}=s_{3}=s_{4}=0 .
\end{aligned}
$$

The corresponding collineation $K^{*}: P_{5}^{*} \rightarrow P_{5}^{\prime *}$ realizing this deformation is given by

$$
\begin{gathered}
K^{*} E_{5}=\sigma E_{5}^{\prime}, \quad K^{*} E_{6}=\sigma^{-1} E_{6}^{\prime}, \quad K^{*} E_{3}=\sigma E_{3}^{\prime}, \quad K^{*} E_{4}=\sigma^{-1} E_{4}^{\prime}, \\
K^{*} E_{1}=\alpha_{15}^{*} E_{5}^{\prime}+\sigma E_{1}^{\prime} ; \quad K^{*} E_{2}=\alpha_{26}^{*} E_{6}^{\prime}+\sigma^{-1} E_{2}^{\prime},
\end{gathered}
$$


where

$$
3 \sigma^{-1} \alpha_{15}^{*} \omega_{1}=2 \tau_{53}-\tau_{31}, \quad 3 \sigma \alpha_{26}^{*} \omega_{2}=2 \tau_{64}-\tau_{42} .
$$

Since the conditions for the correspondence $C^{*}$ to be a projective deformation of the third order are the same as those obtained for the correspondence $C$, we have

Proposition 3. Let $C: L \rightarrow L^{\prime}$ be a developable correspondence, $C^{*}: L^{*} \rightarrow L^{*}$ the induced developable correspondence. The correspondence $C$ is a projective deformation of the third order if and only if the correspondence $C^{*}$ is the projective deformation of the third order.

According to (7), ( $\left.7^{\prime}\right)$ and (27) the collineation (27) in hyperplanar coordinates is given by the equations

$$
\begin{gathered}
K E_{5}=\varrho^{-1} E_{5}^{\prime}, \quad K E_{6}=\varrho E_{6}^{\prime}, \quad K E_{3}=\varrho^{-1} E_{3}^{\prime}, \quad K E_{4}=\varrho E_{4}^{\prime}, \\
K E_{1}=-\varrho^{-2} \alpha_{51} E_{5}^{\prime}+\varrho^{-1} E_{1}^{\prime}, \quad K E_{2}=-\varrho^{2} \alpha_{62} E_{6}^{\prime}+\varrho E_{2}^{\prime}
\end{gathered}
$$

and (28). Comparing (30) and (28) with (29) and (30) we can see that the collineations $K, K^{*}$ are different in general. The case of identifying $K$ and $K^{*}$ will be taken in our considerations in Sec. 14.

\section{RELATIONS OF THE PROJECTIVE DEFORMATION OF CONGRUENCES TO PROJECTIVE DEFORMATIONS OF THEIR FOCAL SURFACES}

The correspondences $C: L \rightarrow L^{\prime}, C^{*}: L^{*} \rightarrow L^{*}$ induce in a known manner correspondences between focal surfaces, which we denote $c_{1}:\left(A_{1}\right) \rightarrow\left(A_{1}^{\prime}\right), c_{2}:\left(A_{2}\right) \rightarrow$ $\rightarrow\left(A_{2}^{\prime}\right), c_{1}^{*}:\left(E_{5}\right) \rightarrow\left(E_{5}^{\prime}\right), c_{2}^{*}:\left(E_{6}\right) \rightarrow\left(E_{6}^{\prime}\right)$. If $C$ is a deformation of the third order, then the correspondences $c_{1}, c_{2}, c_{1}^{*}, c_{2}^{*}$ are simultaneously deformations of the second order, which can be realized by the collineation (27). However, a more general assertion is valid, which we formulate in

Proposition 4. Let $C: L \rightarrow L^{\prime}$ be a correspondence, let $c_{1}:\left(A_{1}\right) \rightarrow\left(A_{1}^{\prime}\right), c_{2}:\left(A_{2}\right) \rightarrow$ $\rightarrow\left(A_{2}^{\prime}\right), c_{1}^{*}:\left(E_{5}\right) \rightarrow\left(E_{5}^{\prime}\right), c_{2}^{*}:\left(E_{6}\right) \rightarrow\left(E_{6}^{\prime}\right)$ be the induced correspondences. The correspondence $C$ is a projective deformation of the third order if and only if $c_{1}$, $c_{2}, c_{1}^{*}, c_{2}^{*}$ are projective deformations of the second order realizable by a common collineation.

Proof. Let us suppose that $C$ is a projective deformation of the third order and $K$ the collineation (27) realizing this deformation. Due to fundamental equations of congruences $L, L^{\prime}$, the conditions (26) and collineation (27), we get

$$
\begin{aligned}
& K A_{1}=\varrho A_{1}^{\prime}, \quad K \mathrm{~d} A_{1}-\varrho \mathrm{d} A_{1}^{\prime}=-\varrho \tau_{11} A_{1}^{\prime} \\
& K \mathrm{~d}^{2} A_{1}-\varrho \mathrm{d}^{2} A_{1}^{\prime}+2 \varrho \tau_{11} \mathrm{~d} A_{1}^{\prime}=0\left(\bmod A_{1}^{\prime}\right)
\end{aligned}
$$


and similar equations are obtained for the remaining pairs of focal surfaces being under consideration. This means that $c_{1}, c_{2}, c_{2}^{*}, c_{1}^{*}$ are projective deformations of the second order realized simultaneously by the collineation $K$.

Conversely let us suppose that $c_{1}, c_{2}, c_{1}^{*}, c_{2}^{*}$ are correspondences induced by a correspondence $C$. From the conditions for $c_{1}$ to be a projective deformation of the second order, i.e.,

$$
\begin{aligned}
& H_{1} A_{1}=\varrho A_{1}^{\prime}, \quad H_{1} \mathrm{~d} A_{1}-\varrho \mathrm{d} A_{1}^{\prime}-\vartheta_{1} A_{1}^{\prime}=0 \\
& H_{1} \mathrm{~d}^{2} A_{1}-\varrho \mathrm{d}^{2} A_{1}^{\prime}-2 \vartheta_{1} \mathrm{~d} A_{1}^{\prime}=0\left(\bmod A_{1}^{\prime}\right),
\end{aligned}
$$

we get that $c_{1}$ necessarily transfers the conjugate net on $\left(A_{1}\right)$ into the conjugate net on $\left(A_{1}^{\prime}\right)$, i.e. $C$ is developable. The most general collineation $H_{1}$ realizing this deformation is given by the equations

$$
\begin{aligned}
& H_{1} A_{1}=\varrho A_{1}^{\prime}, \quad H_{1} A_{2}=\varrho \frac{\alpha_{1}^{\prime}}{\alpha_{1}} A_{2}^{\prime}, \quad H_{1} A_{3}=\varrho A_{3}^{\prime}, \\
& H_{1} A_{4}=\alpha_{41} A_{1}^{\prime}+\alpha_{42} A_{2}^{\prime}+\varrho \frac{\alpha_{1}^{\prime}}{\alpha_{1}} A_{4}^{\prime}, \quad H_{1} A_{5}=\alpha_{51} A_{1}^{\prime}+\alpha_{53} A_{3}^{\prime}+\varrho A_{5}^{\prime} \\
& H_{1} A_{6}=\sum_{j=1}^{6} \alpha_{6 j} A_{j}^{\prime} ; \quad \alpha_{42}=\varrho_{m_{1}}, \quad \alpha_{53}=\varrho s_{1} .
\end{aligned}
$$

Using the following substitution

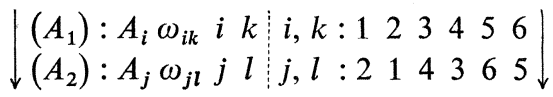

we obtain the most general collineation $\mathrm{H}_{2}$ realizing the deformation of the second order of focal surfaces $\left(A_{2}\right),\left(A_{2}^{\prime}\right)$. Similarly, using the substitutions (10) and (11), we obtain collineations $H_{1}^{*}, H_{2}^{*}$ realizing the deformations of the second order $c_{1}^{*}, c_{2}^{*}$. From the conditions $H_{1} \equiv H_{2} \equiv H_{1}^{*} \equiv H_{2}^{*}$ we get easily the relations (26), i.e. $C$ is a projective deformation of the third order. Our Proposition is proved.

\section{SOME OTHER GEOMETRICAL MEANINGS OF A PROJECTIVE DEFORMATION OF THE THIRD ORDER}

Proposition 5. A correspondence $C: L \rightarrow L^{\prime}$ is a projective deformation of the third order if and only if there exists a collineation $K$ realizing simultaneously deformations of the second order $C: L \rightarrow L^{\prime}, c_{1}:\left(A_{1}\right) \rightarrow\left(A_{1}^{\prime}\right), c_{1}^{*}:\left(E_{5}\right) \rightarrow\left(E_{5}^{\prime}\right)$.

Proof. If the correspondence $C$ is a projective deformation of the third order, then collineation (27) realizing this deformation realizes the deformation of the second order $C: L \rightarrow L^{\prime}$, too, and according to Proposition 4, also the deformations of the second order $c_{1}:\left(A_{1}\right) \rightarrow\left(A_{1}^{\prime}\right), c_{1}^{*}:\left(E_{5}\right) \rightarrow\left(E_{5}^{\prime}\right)$. In order to prove the second 
part of the assertion, observe the fact that the most general collineation realizing simultaneously the deformations of the second order $C, c_{1}$ and the corresponding analytical conditions can be obtained by simultanous fulfilling of conditions (20), (21), (22), (32). We have

$$
\begin{gathered}
K A_{1}=\varrho A_{1}^{\prime}, \quad K A_{2}=\varrho^{-1} A_{2}^{\prime}, \quad K A_{3}=\varrho A_{3}^{\prime}, \quad K A_{4}=\varrho^{-1} A_{4}^{\prime}, \\
K A_{5}=\alpha_{51} A_{1}^{\prime}+\alpha_{53} A_{3}^{\prime}+\varrho A_{5}^{\prime}, \quad K A_{6}=\alpha_{61} A_{1}^{\prime}+\alpha_{62} A_{2}^{\prime}+\alpha_{64} A_{4}^{\prime}+\varrho^{-1} A_{6}^{\prime}, \\
\alpha_{53}=\varrho s_{1}, \quad \alpha_{64}=\varrho^{-1} s_{2} ; \quad \alpha_{1}^{\prime}=\varrho^{-2} \alpha_{1}, \quad \alpha_{2}^{\prime}=\varrho^{2} \alpha_{2} .
\end{gathered}
$$

Turning the collineation (32) into the dual form and comparing it with the collineation $K$ in hyperplanar coordinates, we find the necessary and sufficient conditions for projective deformations of the second order $C, c_{1}, c_{1}^{*}$ to be simultaneously realized by the same collineation in the form

$$
\alpha_{1}^{\prime}=\varrho^{-2} \alpha_{1}, \quad \alpha_{2}^{\prime}=\varrho^{2} \alpha_{2}, \quad \beta_{1}^{\prime}=\varrho^{-2} \beta_{1}, \quad s_{1}=s_{3}=0, \quad n_{1}=\varrho^{2} s_{2} .
$$

These conditions are equivalent to (27) as it follows from (15) and (16).

A. Švec has proved in the paper [1] that a projective deformation of the second order of congruences $L, L^{\prime}$ in $P_{n}, n \geqq 5$, is equivalent to their point deformation. However, the osculating collineations of considered deformations are different in general. The same holds also for dual congruences introduced in Sec. 2. The following Proposition deals with a special case that three of the mentioned deformations are realized by the same collineation.

Proposition 6. Let collineation $K$ realize a projective deformation of the second order of $L, L^{\prime}$; collineation $K^{*}$ realize a projective deformation of the second order of $L^{*}, L^{\prime *}$; collineation $H$ realize a point deformation of congruences $L, L^{\prime}$; collineation $H^{*}$ realize a point deformation of congruences $L^{*}, L^{\prime *}$. The congruences $L, L^{\prime}$ are in projective deformation of the third order if and only if at least three collineations from $\mathrm{K}, \mathrm{K}^{*}, \mathrm{H}, \mathrm{H}^{*}$, realizing simultaneously the three corresponding deformations, are identifying.

Proof. According to [3] (p. 16, 17), and with respect to our specialization of frames, the most general collineation realizing the point deformation and the corresponding analytical conditions are

$$
\begin{gathered}
H A_{1}=\pi_{11} A_{1}^{\prime}, \quad H A_{2}=\pi_{22} A_{2}^{\prime}, \quad H A_{3}=\pi_{11} A_{3}^{\prime}, \quad H A_{4}=\pi_{22} A_{4}^{\prime}, \\
H A_{5}=\sum_{j=1}^{6} \pi_{5 j} A_{j}^{\prime}, \quad H A_{6}=\sum_{j=1}^{6} \pi_{6 j} A_{j}^{\prime}, \\
\pi_{11} \alpha_{2}=\pi_{22} \alpha_{2}^{\prime}, \quad \pi_{22} \alpha_{1}=\pi_{11} \alpha_{1}^{\prime} .
\end{gathered}
$$

By means of substitutions (10), (11), (17) in (21), (22), (23), we get the collineations $K^{*}$, $H^{*}$ and the corresponding analytical conditions. Now it is clear that if $C$ is a projec- 
tive deformation of the third order, then the collineation (27), (28) realizes all considered deformations. The converse can be easily proved if we identify any of the three mentioned collineations and take the corresponding analytical conditions into consideration.

\section{EXISTENCE THEOREM}

Let $C: L \rightarrow L^{\prime}$ be a projective deformation of the third order. Without loss of generality we may put $\varrho=1$ in (26), (27), (28). Consequently, the system (3), (4), $\left(3^{\prime}\right),\left(4^{\prime}\right),(12),(13),(14),(15),(16)$ can be arranged so that the triplet $\left(C, L, L^{\prime}\right)$ is given by the closed system (3), (4) and

$$
\begin{gathered}
\tau_{14}=\tau_{15}=\tau_{16}=\tau_{23}=\tau_{25}=\tau_{26}=\tau_{32}=\tau_{34}=\tau_{36}=0, \\
\tau_{41}=\tau_{43}=\tau_{45}=\tau_{54}=\tau_{63}=\tau_{13}=\tau_{24}=\tau_{12}=\tau_{21}=0, \\
\tau_{56}=\tau_{65}=\tau_{11}=\tau_{22}=\tau_{33}=\tau_{44}=\tau_{55}=\tau_{66}=0, \\
\omega_{1} \wedge \tau_{31}=0, \quad \omega_{1} \wedge \tau_{53}=0, \quad \omega_{2} \wedge \tau_{42}=0, \omega_{2} \wedge \tau_{64}=0, \\
\beta_{2} \omega_{1} \wedge \tau_{64}-\omega_{2} \wedge \tau_{52}=0, \quad \alpha_{2} \omega_{1} \wedge \tau_{42}-\omega_{2} \wedge \tau_{61}=0, \\
\omega_{1} \wedge \tau_{52}-\alpha_{1} \omega_{2} \wedge \tau_{31}=0, \quad \omega_{1} \wedge \tau_{61}-\beta_{1} \omega_{2} \wedge \tau_{53}=0 .
\end{gathered}
$$

The system is not involutive, so we augment it by equations

$$
\begin{array}{ll}
\tau_{31}=\bar{g}_{2} \omega_{1}, \quad \tau_{53}=\bar{h}_{2} \omega_{1}, & \tau_{42}=\bar{h}_{1} \omega_{2}, \quad \tau_{64}=\bar{g}_{1} \omega_{2}, \\
\tau_{52}=-\beta_{2} \bar{g}_{1} \omega_{1}-\alpha_{1} \bar{g}_{2} \omega_{2}, & \tau_{61}=-\alpha_{2} \bar{h}_{1} \omega_{1}-\beta_{1} \bar{h}_{2} \omega_{2} .
\end{array}
$$

Using exterior differentiation of (35) and equations (4), we get

$$
\begin{aligned}
\omega_{1} & \wedge\left\{\mathrm{d} \bar{g}_{2}-\bar{g}_{2}\left(\omega_{55}-\omega_{11}\right)+\tau_{51}\right\}=0, \\
\omega_{1} & \wedge\left\{\mathrm{d} \bar{h}_{2}-\bar{h}_{2}\left(\omega_{55}-\omega_{11}\right)-\tau_{51}\right\}=0, \\
\omega_{2} & \wedge\left\{\mathrm{d} \bar{h}_{1}-\bar{h}_{1}\left(\omega_{66}-\omega_{22}\right)+\tau_{62}\right\}=0, \\
\omega_{2} & \wedge\left\{\mathrm{d} \bar{g}_{1}-\bar{g}_{1}\left(\omega_{66}-\omega_{22}\right)-\tau_{62}\right\}=0, \\
\beta_{2} \omega_{1} & \wedge\left\{\mathrm{d} \bar{g}_{1}-\bar{g}_{1}\left(\omega_{66}-\omega_{22}\right)-\tau_{62}\right\}+ \\
+\alpha_{1} \omega_{2} & \wedge\left\{\mathrm{d} \bar{g}_{2}-\bar{g}_{2}\left(\omega_{55}-\omega_{11}\right)+\tau_{51}\right\}=0, \\
\alpha_{2} \omega_{1} & \wedge\left\{\mathrm{d} \bar{h}_{1}-\bar{h}_{1}\left(\omega_{66}-\omega_{22}\right)+\tau_{62}\right\}+ \\
+\beta_{1} \omega_{2} & \wedge\left\{\mathrm{d} \bar{h}_{2}-\bar{h}_{2}\left(\omega_{55}-\omega_{11}\right)-\tau_{51}\right\}=0 .
\end{aligned}
$$

The augmented system is not involutive yet, so we make a further augmentation of (36),

(37) $\mathrm{d} \bar{g}_{2}-\bar{g}_{2}\left(\omega_{55}-\omega_{11}\right)+\tau_{51}=\beta_{2} r \omega_{1}, \mathrm{~d} \bar{h}_{2}-\bar{h}_{2}\left(\omega_{55}-\omega_{11}\right)-\tau_{51}=\alpha_{2} s \omega_{1}$,

$$
\mathrm{d} \bar{h}_{1}-\bar{h}_{1}\left(\omega_{66}-\omega_{22}\right)+\tau_{62}=\beta_{1} s \omega_{2}, \mathrm{~d} \bar{g}_{1}-\bar{g}_{1}\left(\omega_{66}-\omega_{22}\right)-\tau_{62}=\alpha_{1} r \omega_{2} \text {. }
$$


Using equations (4) we can arrange the exterior differentials of (37) so that they take the form

$$
\begin{gathered}
\beta_{2} \omega_{1} \wedge\left\{\mathrm{d} r-r\left(\omega_{66}-\omega_{11}\right)\right\}+ \\
+\omega_{1} \wedge \omega_{31}\left(\bar{g}_{2}+\bar{h}_{2}\right)-\left(\bar{g}_{2}+\bar{h}_{2}\right) \beta_{1} \beta_{2} \omega_{1} \wedge \omega_{2}=0, \\
\alpha_{2} \omega_{1} \wedge\left\{\mathrm{d} s-s\left(\omega_{55}-\omega_{22}\right)\right\}+ \\
+\omega_{1} \wedge \omega_{53}\left(\bar{g}_{2}+\bar{h}_{2}\right)-\left(\bar{g}_{2}+\bar{h}_{2}\right) \alpha_{1} \alpha_{2} \omega_{1} \wedge \omega_{2}=0, \\
\beta_{1} \omega_{2} \wedge\left\{\mathrm{d} s-s\left(\omega_{55}-\omega_{22}\right)\right\}+ \\
+\omega_{2} \wedge \omega_{42}\left(\bar{g}_{1}+\bar{h}_{1}\right)+\left(\bar{g}_{1}+\bar{h}_{1}\right) \beta_{1} \beta_{2} \omega_{1} \wedge \omega_{2}=0, \\
\alpha_{1} \omega_{2} \wedge\left\{\mathrm{d} r-r\left(\omega_{66}-\omega_{11}\right)\right\}+ \\
+\omega_{2} \wedge \omega_{64}\left(\bar{g}_{1}+\bar{h}_{1}\right)+\left(\bar{g}_{1}+\bar{h}_{1}\right) \alpha_{1} \alpha_{2} \omega_{1} \wedge \omega_{2}=0 .
\end{gathered}
$$

If $\left(\bar{g}_{1}+\bar{h}_{1}\right)$ and $\left(\bar{g}_{2}+\bar{h}_{2}\right)$ are not simultaneously equal to zero, the rank of the polar matrix is 14 , which is the number of linearly independent exterior quadratic relations. The system is involutive and we have the result

Proposition 7. The triplets $\left(C, L, L^{\prime}\right)$, where $C: L \rightarrow L^{\prime}$ is a projective deformation of the third order, exist and depend on 14 functions of one argument.

The case that both $\left(\bar{g}_{1}+\bar{h}_{1}\right)$ and $\left(\bar{g}_{2}+\bar{h}_{2}\right)$ are equal to zero will be dealt with in Sec. 13.

\section{TOTALLY QUASIASYMPTOTIC CORRESPONDENCE}

Let $C: L \rightarrow L^{\prime}$ be an arbitrary developable correspondence. Let $C^{b}$ be its point extension given by projectivity $\pi$ between straight lines $p \in L$ and $p^{\prime} \in L^{\prime}$ such that the foci on $p^{\prime}$ are attached to foci on $p$, i.e.

$$
\pi A_{1}=\varrho A_{1}^{\prime}, \quad \pi A_{2}=\sigma A_{2}^{\prime} .
$$

By means of $C$ a non-developable ruled surface $R^{\prime}=C R$ in $L^{\prime}$ corresponds to a nondevelopable ruled surface $R$ in $L$.

A point $X=x_{1} A_{1}+x_{2} A_{2}$ describes a quasiasymptotic curve on $R$ if and only if it fulfils the condition

$$
\left[A_{1}, A_{2}, \mathrm{~d} A_{1}, \mathrm{~d} A_{2}, \mathrm{~d}^{2} X, \mathrm{~d}^{3} X\right]=0
$$

(see [2] p. 93.). The fundamental equations (2), (3) allow us to express the equation of the quasiasymptotic curve on $R$ passing through the point $X$ in the form

$$
\begin{gathered}
3 \omega_{1}^{2} \omega_{2}^{2}\left(x_{1} \mathrm{~d} x_{2}-x_{2} \mathrm{~d} x_{1}\right)+x_{1}^{2}\left(\alpha_{1} \omega_{2}^{3}+\beta_{2} \omega_{1}^{3}\right) \omega_{1}^{2}-x_{2}^{2}\left(\alpha_{2} \omega_{1}^{3}+\beta_{1} \omega_{2}^{3}\right) \omega_{2}^{2}+ \\
+3 x_{1} x_{2}\left(\omega_{1} \mathrm{~d} \omega_{2}-\omega_{2} \mathrm{~d} \omega_{1}\right) \omega_{1} \omega_{2}+ \\
+x_{1} x_{2}\left(\omega_{22}+\omega_{44}+\omega_{66}-\omega_{11}-\omega_{33}-\omega_{55}\right) \omega_{1}^{2} \omega_{2}^{2}=0
\end{gathered} .
$$


and analogously we get the condition for the point $X^{\prime}=x_{1} \varrho A_{1}^{\prime}+x_{2} \sigma A_{2}^{\prime}$ to describe the quasiasymptotic curve on the ruled surface $R^{\prime}=C R$. The quasiasymptotic passing through points $X, X^{\prime}$ correspond to each other in extension (39) if and only if

$$
\begin{gathered}
x_{1}^{2}\left\{\left(\alpha_{1}-\frac{\varrho}{\sigma} \alpha_{1}^{\prime}\right) \omega_{2}^{3}+\left(\beta_{2}-\frac{\varrho}{\sigma} \beta_{2}^{\prime}\right) \omega_{1}^{3}\right\} \omega_{1}^{2}- \\
-x_{2}^{2}\left\{\left(\alpha_{2}-\frac{\sigma}{\varrho} \alpha_{2}^{\prime}\right) \omega_{1}^{3}+\left(\beta_{1}-\frac{\sigma}{\varrho} \beta_{1}^{\prime}\right) \omega_{2}^{3}\right\} \omega_{2}^{2}+ \\
+x_{1} x_{2}\left\{3 \mathrm{~d} \lg \frac{\varrho}{\sigma}+\tau_{11}+\tau_{33}+\tau_{55}-\tau_{22}-\tau_{44}-\tau_{66}\right\} \omega_{1} \omega_{2}=0 .
\end{gathered}
$$

The extension (39) is the quasiasymptotic correspondence $R \rightarrow R^{\prime}$ if and only if (40) is satisfied identically in $x_{1}, x_{2}$ on $R$, i.e.

$$
\begin{gathered}
\left(\sigma \alpha_{1}-\varrho \alpha_{1}^{\prime}\right) \omega_{2}^{3}+\left(\sigma \beta_{2}-\varrho \beta_{2}^{\prime}\right) \omega_{1}^{3}=0, \\
\left(\varrho \alpha_{2}-\sigma \alpha_{2}^{\prime}\right) \omega_{1}^{3}+\left(\varrho \beta_{1}-\sigma \beta_{1}^{\prime}\right) \omega_{2}^{3}=0, \\
3 \mathrm{~d} \lg \frac{\varrho}{\sigma}+\tau_{11}+\tau_{33}+\tau_{55}-\tau_{22}-\tau_{44}-\tau_{66}=0 .
\end{gathered}
$$

Proposition 8. Let $C: L \rightarrow L^{\prime}$ and $C^{*}: L^{*} \rightarrow L^{*}$ be a projective bideformation of the second order, $C^{b}$ the point extension of $C$, and $R$ a nondevelopable ruled surface in $L$. The correspondence $C^{b}: R \rightarrow C R$ is quasiasymptotic if and only if on $R$,

$$
s_{1} \omega_{1}-s_{2} \omega_{2}=0 \text {. }
$$

Proof. According to A. Švec ([2] p. 95) the congruences $L, L^{\prime}$ are in projective bideformation of the second order if projective deformations of the second order $C: L \rightarrow L^{\prime}$ and $C^{*}: L^{*} \rightarrow L^{*}$ are realized by a common collineation. The necessary and sufficient condition for this is the equality of forms $\varphi=\varphi^{\prime}, \varphi^{*}=\varphi^{*}, F_{1}=F_{1}^{\prime}$. Then the first two of conditions (41) are fulfilled and the last one is reduced to $s_{1} \omega_{1}-s_{2} \omega_{2}=0$ as it is clear from (16), where $m_{1}=m_{2}=n_{1}=n_{2}=0$.

Definition 2. Let $C: L \rightarrow L^{\prime}$ be a developable correspondence, $C^{\boldsymbol{b}}$ be its point extension. According to E. СЕCH we shall call the correspondence $C$ totally quasiasymptotic, if $C^{b}: R \rightarrow C R$ is the quasiasymptotic correspondence for every nondevelopable ruled surface in $L$.

Proposition 9. A developable correspondence $C: L \rightarrow L^{\prime}$ is the projective deformation of the third order if and only if $C$ is totally quasiasymptotic.

Proof. Let $C$ be a projective deformation of the third order. Then the conditions (41) are fulfilled for every non-developable surface $R$ in $L$. Conversely, let $C$ be totally quasiasymptotic. Then (41) must be satisfied identically and the consequences of that fact yield conditions equivalent to (26). 


\section{DEFORMATION OF LAPLACE TRANSFORMS}

A correspondence $C: L \rightarrow L^{\prime}$ induces correspondences $C_{1}: L_{1} \rightarrow L_{1}^{\prime}$ and $C_{-1}$ : $: L_{-1} \rightarrow L_{-1}^{\prime}$ between Laplace transforms of congruences $L, L^{\prime}$. In the paper [4] the necessary and sufficient conditions for correspondences $C, C_{1}, C_{-1}$ to be projective deformations of the second order were found and the cases that two or three of their realizing collineations identify each other were considered. This section is devoted to the investigation of relations between the above mentioned cases and the projective deformation of the third order. For these reasons, let us express the principal forms $\omega_{31}, \omega_{42}, \omega_{53}, \omega_{64}$ from the equations (4).

$$
\begin{array}{ll}
\omega_{31}=g_{2} \omega_{1}+a_{1} \omega_{1}, & \omega_{42}=a_{2} \omega_{1}+h_{1} \omega_{2}, \\
\omega_{53}=h_{2} \omega_{1}+b_{1} \omega_{2}, & \omega_{64}=b_{2} \omega_{1}+g_{1} \omega_{2} .
\end{array}
$$

The point form $\varphi_{1}$ of the Laplace transform of $L$ in the direction $\omega_{2}=0$ is the cross-ratio of the poins

$$
A_{1}, A_{3},\left(A_{1}+\mathrm{d} A_{1}\right)_{\omega_{2}=0},\left(A_{3}+\mathrm{d} A_{3}\right)_{\omega_{1}=0},
$$

and an analogous statement holds for point forms $\varphi_{-1}, \varphi_{1}^{*}, \varphi_{-1}^{*}$ of the Laplace transforms $L_{-1}$ and $L_{1}^{*}, L_{-1}^{*}$ of congruences $L$ or $L^{*}$, respectively. Using the fundamental equations, we get the point forms of congruences $L_{1}, L_{-1}, L_{1}^{*}, L_{-1}^{*}$, which are succesively

$$
\begin{array}{ll}
\varphi_{1}=a_{1} \omega_{1} \omega_{2}, & \varphi_{-1}=a_{2} \omega_{1} \omega_{2}, \\
\varphi_{1}^{*}=b_{1} \omega_{1} \omega_{2}, & \varphi_{-1}^{*}=b_{2} \omega_{1} \omega_{2} .
\end{array}
$$

Under condition that $C: L \rightarrow L^{\prime}$ is a projective deformation of the third order the equations (42), (42') and (35) yield

$$
a_{1}^{\prime}=a_{1}, \quad a_{2}^{\prime}=a_{2}, \quad b_{1}^{\prime}=b_{1}, \quad b_{2}^{\prime}=b_{2}
$$

and with respet to $(43),\left(43^{\prime}\right)$ we have

$$
\varphi_{1}^{\prime}=\varphi_{1}, \quad \varphi_{-1}^{\prime}=\varphi_{-1}, \quad \varphi_{1}^{\prime *}=\varphi_{1}^{*}, \quad \varphi_{-1}^{* *}=\varphi_{-1}^{*} .
$$

We can formulate these results in

Proposition 10. Let $C: L \rightarrow L^{\prime}$ be a correspondence, $C_{1}: L_{1} \rightarrow L_{1}^{\prime}, C_{-1}: L_{-1} \rightarrow$ $\rightarrow L_{-1}^{\prime}, C_{1}^{*}: L_{1}^{*} \rightarrow L_{1}^{\prime *}, C_{-1}^{*}: L_{-1}^{*} \rightarrow L_{-1}^{*}$ the induced correspondences. If $C$ is a projective deformation of the third order, then the correspondences $C_{1}, C_{-1}, C_{1}^{*}$, $C_{-1}^{*}$ are projective deformations of second order.

Comparing the Proposition 1 in this paper with the Proposition 2 in [4] we obtain 
Proposition 11. A correspondence $C: L \rightarrow L^{\prime}$ is a projective deformation of the third order if and only if projective deformations of the second order $C: L \rightarrow L^{\prime}$, $C_{1}: L_{1} \rightarrow L_{1}^{\prime}$ or $C: L \rightarrow L^{\prime}, C_{-1}: L_{-1} \rightarrow L_{-1}^{\prime}$ are realized by a common osculating collineation.

As shown in [4], Proposition 4, if projective deformations of second order $C$, $C_{1}\left(C, C_{-1}\right)$ are realized simultaneously by a common osculating collineation $H_{1}\left(H_{0}\right)$, then there exists a collineation $H_{0}\left(H_{1}\right)$ realizing simultaneously the deformations of the second order $C, C_{-1}\left(C, C_{1}\right)$. The collineations $H_{1}, H_{0}$ are different in general. Repeating this process we arrive at the result that the same holds for every pair of induced correspondences $C_{i}, C_{i+1}(i= \pm 1, \pm 2, \ldots)$. From this fact and from Proposition 11 it follows,

Proposition 12. Let $\ldots, L_{-1}, L, L_{1}, \ldots ; \ldots L_{-1}^{\prime}, L^{\prime}, L_{1}^{\prime}, \ldots$ be Laplace successions of congruences in $P_{5}$ or $P_{5}^{\prime}$ respectively. Let $C: L \rightarrow L^{\prime}$ be a correspondence and $C_{i}: L_{i} \rightarrow L_{i}^{\prime}(i= \pm 1, \pm 2, \ldots)$ the induced correspondences. If $C$ is a projective deformation of the third order, then every $C_{i}$ is a projective deformation of the third order.

Let us remark that analogous assertions hold for dual congruences, too, as it is apparent from Proposition 3.

\section{SINGULAR PROJECTIVE DEFORMATION OF THE THIRD ORDER}

Let us assume that the congruences $L, L^{\prime}$ are in a projective deformation of the third order and choose the frames $R, R^{\prime}$ such that $\alpha_{1}^{\prime}=\alpha_{1}$. Then the equations (34), (35) hold. If $K$ is the collineation (27), (28), we have

$$
\begin{gathered}
K A_{1}=A_{1}^{\prime}, K \mathrm{~d} A_{1}=\mathrm{d} A_{1}^{\prime}, \\
K \mathrm{~d}^{2} A_{1}=\mathrm{d}^{2} A_{1}^{\prime}-\frac{1}{3}\left(\bar{g}_{2}+\bar{h}_{2}\right) \omega_{1}^{2} A_{1}^{\prime}, \\
K \mathrm{~d}^{3} A_{1}=\mathrm{d}_{3} A_{1}^{\prime}-\left(\bar{g}_{2}+\bar{h}_{2}\right) \omega_{1}^{2} \mathrm{~d} A_{1}^{\prime}-\left\{\left[\mathrm{d} \bar{g}_{2}-\bar{g}_{2}\left(\omega_{55}-\omega_{11}\right)+\tau_{51}\right] \omega_{1}^{2}+\right. \\
\left.+\frac{1}{3}\left(\bar{g}_{2}+\bar{h}_{2}\right)\left[3 \mathrm{~d} \omega_{1}-\omega_{1}\left(2 \omega_{11}-\omega_{33}-\omega_{55}\right)\right]\right\} A_{1}^{\prime}+ \\
+\frac{1}{3}\left\{2 \beta_{2}\left(\bar{g}_{1}+\bar{h}_{1}\right) \omega_{1}^{3}+3 \alpha_{1}\left(\bar{g}_{2}+\bar{h}_{2}\right) \omega_{1}^{2} \omega_{2}-2 \alpha_{1}\left(\bar{g}_{1}+\bar{h}_{1}\right) \omega_{2}^{3}\right\} A_{2}^{\prime} .
\end{gathered}
$$

From this we obtain at once

Proposition 13. Let $C: L \rightarrow L^{\prime}$ be a projective deformation of the third order and $K$ be its realizing collineation. The induced correspondence $c_{1}:\left(A_{1}\right) \rightarrow\left(A_{1}^{\prime}\right)$ is a projective deformation of the third order realized by the collineation $K$ if and only if

$$
\bar{g}_{1}+\bar{h}_{1}=0, \quad \bar{g}_{2}+\bar{h}_{2}=0 .
$$


Similarly we can get the same conditions (45) for the correspondence $c_{2}:\left(A_{2}\right) \rightarrow$ $\rightarrow\left(A_{2}^{\prime}\right)$ so that we have

Proposition 14. Let $C: L \rightarrow L^{\prime}$ be a projective deformation of the third order and $K$ its realizing collineation. If the induced correspondence $c_{1}:\left(A_{1}\right) \rightarrow\left(A_{1}^{\prime}\right)$ is a projective deformation of the third order realized by the collineation $K$, then $c_{2}$ : $:\left(A_{2}\right) \rightarrow\left(A_{2}^{\prime}\right)$ is also a projective deformation of the third order realized by the collineation $K$ and vice versa.

In accordance with E. Čech and E. CARTAN we define now the singular deformation.

Definition 3. Let $C: L \rightarrow L^{\prime}$ be a projective deformation of the third order and $K$ its realizing osculating collineation. We shall say that $C$ is singular if both induced correspondences $c_{1}:\left(A_{1}\right) \rightarrow\left(A_{1}^{\prime}\right)$ and $c_{2}:\left(A_{2}\right) \rightarrow\left(A_{2}^{\prime}\right)$ are projective deformations of the third order and if they can be realized by the same collineation $K$.

\section{EXISTENCE OF A SINGULAR DEFORMATION}

Observe first that the singular projective deformation of the third order is the case excluded in Proposition 7. In order to find out the existence of these deformations, let us use conditions (45) in (36). It follows immediately

$$
\begin{aligned}
& \mathrm{d} \bar{g}_{2}-\bar{g}_{2}\left(\omega_{55}-\omega_{11}\right)+\tau_{51}=p_{2} \omega_{1}, \\
& \mathrm{~d} \bar{h}_{1}-\bar{h}_{1}\left(\omega_{66}-\omega_{22}\right)+\tau_{62}=p_{1} \omega_{2},
\end{aligned}
$$

where $p_{1}=p_{2}=0$ by $\alpha_{1} \alpha_{2}-\beta_{1} \beta_{2} \neq 0$; then it can be easily checked that congruences $L, L^{\prime}$ are identical.

Therefore, we shall suppose in the following that

$$
\alpha_{1} \alpha_{2}-\beta_{1} \beta_{2}=0
$$

i.e., both congruences $L$ and $L^{\prime}$ are necessarily W-congruences. Now it results from the systems (36), (45) that

$$
p_{1}=\alpha_{1} f, \quad p_{2}=-\beta_{2} f
$$

in (46), and performing the exterior differentiation we find out with respect to (4) that

$$
\mathrm{d} f-f\left(\omega_{66}-\omega_{11}\right)=0 .
$$


If $f=0$ then both $L$ and $L^{\prime}$ are again identical, so we shall suppose $f \neq 0$. From the system (4) and (47) we obtain

$$
\mathrm{d} \lg \frac{\beta_{2}}{\alpha_{2}}=\mathrm{d} \lg \frac{\alpha_{1}}{\beta_{1}}=\omega_{11}-\omega_{22}+\omega_{55}-\omega_{66} .
$$

Using exterior differentiation of (49) and (50) we find out that in (42) we necessarily have

$$
a_{1}=b_{2}, \quad b_{1}=a_{2}
$$

i.e. the quasiasymptotic curves are in correspondence on focal surfaces $\left(A_{1}\right),\left(A_{3}\right)$ or $\left(A_{2}\right),\left(A_{4}\right)$ respectively, as it is clear from their equations

$$
\begin{aligned}
& \beta_{2} \omega_{1}^{3}+\alpha_{1} \omega_{2}^{3}=0, \quad \alpha_{2} a_{2} \omega_{1}^{3}+\beta_{1} b_{1} \omega_{2}^{3}=0, \\
& \alpha_{2} \omega_{1}^{3}+\beta_{1} \omega_{2}^{3}=0, \quad \beta_{2} b_{2} \omega_{1}^{3}+\alpha_{1} a_{1} \omega_{2}^{3}=0,
\end{aligned}
$$

which can be computed from the condition that their 3-osculating space is obtained in 2-osculating space of the considered surface. Hence both congruences $L, L^{\prime}$ are R-congruences as it follows from (42), (51), (35) and [3] Proposition 20.

Proposition 15. Let $C: L \rightarrow L^{\prime}$ be a singular projective deformation of the third order. Then both congruences $L, L^{\prime}$ are $\mathrm{R}$-congruences and their focal surfaces are R-surfaces.

If (47) and (51) hold, the congruence $L$ is a general R-congruence and the equation (49) is completely integrable. This and [3] (p. 49) yield

Proposition 16. The triplets $\left(C, L, L^{\prime}\right)$, where $C: L \rightarrow L^{\prime}$ is a singular projective deformation of the third order, exist and depend on ten functions of one argument. The congruence $L$ is a general R-congruence (depending on ten functions of one variable), the congruence $L^{\prime}$ depends on one constant.

Now, we shall prove

Proposition 17. Let $C: L \rightarrow L^{\prime}$ be singular projective deformation realized by a collineation $K$. If the induced correspondences $c_{1}:\left(A_{1}\right) \rightarrow\left(A_{1}^{\prime}\right)$ or $c_{2}:\left(A_{2}\right) \rightarrow\left(A_{2}^{\prime}\right)$ are projective deformations of the fourth order realized by a collineation $K$, then congruences $L, L$ are identical.

Proof. Without loss of generality we may choose $\bar{g}_{2}=\bar{h}_{1}=0$ in equations (46). Then the collineation $K$ is given by equations

$$
K A_{i}=A_{i}^{\prime} \quad(i=1,2, \ldots, 6)
$$


and we can get by a direct computation,

$$
\begin{gathered}
K A_{1}=A_{1}^{\prime}, K \mathrm{~d} A_{1}=\mathrm{d} A_{1}^{\prime}, \quad K \mathrm{~d}^{2} A_{1}=\mathrm{d}^{2} A_{1}^{\prime}, \\
K \mathrm{~d}^{3} A_{1}=\mathrm{d}^{3} A_{1}^{\prime}+\beta_{2} f \omega_{1}^{3} A_{1}^{\prime}, \\
K \mathrm{~d}^{4} A_{1}^{\prime}=\mathrm{d}^{4} A_{1}^{\prime}+4 \beta_{2} f \omega_{1}^{3} \mathrm{~d} A_{1}^{\prime}-\alpha_{1} f \omega_{2}\left(\alpha_{1} \omega_{2}^{3}+4 \beta_{2} \omega_{1}^{3}\right) A_{2}^{\prime}- \\
-3 \beta_{2} f \omega_{1}^{4} A_{3}^{\prime}\left(\bmod A_{1}^{\prime}\right) .
\end{gathered}
$$

If $c_{1}$ is a projective deformation of the fourth order, then necessarily $f=0$ and congruences $L, L^{\prime}$ are identical. Similarly we can get the same result for focal surfaces $\left(A_{2}\right),\left(A_{2}^{\prime}\right)$.

\section{SOME PROPERTIES OF CONGRUENCES IN A SINGULAR DEFORMATION}

According to Propositions 13, 14 and the substitution (10), we have

Proposition 18. A correspondence $C: L \rightarrow L^{\prime}$ is a singular projective deformation of the third order if and only if the induced correspondence $C^{*}: L^{*} \rightarrow L^{*}$ is a singular projective deformation of the third order.

Comparing the collineations $K, K^{*}$ given by (27), (28), (29), (30) and taking the results in Propositions 13, 14 into our consideration, we get

Proposition 19. A correspondence $C: L \rightarrow L^{\prime}$ is a singular projective deformation of the third order if and only if the projective deformations of the third order $C: L \rightarrow L^{\prime}$ and $C^{*}: L^{*} \rightarrow L^{*}$ are realized by a common collineation.

In the paper [4] the necessary and sufficient conditions for projective deformations of the second order $C: L \rightarrow L^{\prime}, C_{1}: L_{1} \rightarrow L_{1}^{\prime}, C_{-1}: L_{-1} \rightarrow L_{-1}^{\prime}$ to be realized by a common collineation were found. It was shown there that these conditions are equivalent to projective deformation of the third order of focal surfaces $\left(A_{1}\right),\left(A_{1}^{\prime}\right)$. A comparison of relative components of frames in [4] with those used in this paper shows that the above mentioned conditions are equivalent to conditions (26) and (47) for $C$ to be a singular deformation of the third order. Hence we have

Proposition 20. A correspondence $C: L \rightarrow L^{\prime}$ is a singular projective deformation of the third order if and only if projective deformations of the second order $C: L \rightarrow$ $\rightarrow L^{\prime}, C_{1}: L_{1} \rightarrow L_{1}^{\prime}, C_{-1}: L_{-1} \rightarrow L_{-1}^{\prime}$ are simultaneously realized by a common collineation.

Proposition 21. A correspondence $C: L \rightarrow L^{\prime}$ is a singular projective deformation of the third order if and only if the induced correspondence $c_{1}:\left(A_{1}\right) \rightarrow\left(A_{1}^{\prime}\right)$ is a projective deformation of the third order. 
If $c_{1}:\left(A_{1}\right) \rightarrow\left(A_{1}^{\prime}\right)$ is a projective deformation of the third order then all induced correspondences $\ldots, c_{-1}, c_{1}, c_{2}, \ldots$ are projective deformations of the third order (see [3] p. 54). Recalling Proposition 21, we have

Proposition 22. Let $\ldots, L_{-1}, L, L_{1} \ldots ; \ldots, L_{-1}^{\prime}, L^{\prime}, L_{1}^{\prime}, \ldots$ be Laplace successions of congruences in $P_{5}$ or $P_{5}^{\prime}$ respectively. Let $C: L \rightarrow L^{\prime}$ be a correspondence and $C_{i}: L_{i} \rightarrow L_{i}^{\prime}(i= \pm 1, \pm 2, \ldots)$ be the induced correspondences. If $C$ is a singular projective deformation of the third order, then all correspondences $C_{i}$ are singular projective deformations of the third order.

\section{References}

[1] A. Švec: Déformation projective des congruences de droites dans $S_{n}$. Czech. Math. Journ., $5(80), 1955,546-558$.

[2] A. Svec: Les congruences de droites dans $S_{5}$. Publ. Fac. Sci. Univ. Masaryk, Brno, No 382, 1957.

[3] A. Svec: Projective Differential Geometry of Line Congruences, Prague 1965.

[4] J. Beneš: Projektivní deformace kongruencí a jejich Laplaceových transformací. Sborník VA AZ, řada B (technická), zvláštní č́slo 1966, 75-84.

Author's address: Brno, Leninova 75, ČSSR (Vojenská akademie Antonína Zápotockého). 